\title{
The Widths of a Polymer Chain. II. Longitudinal Boundary of a Random Coil Chain
}

\author{
Keiji MORO \\ Department of Applied Physics, Waseda University, \\ 3-4-1 Ohkubo, Shinjuku-ku, Tokyo 160, Japan.
}

(Received April 4, 1980)

\begin{abstract}
The width of a Gaussian random-coil chain along the line connecting both ends is calculated by solving an appropriate diffusion equation. First, this parameter is obtained under the restriction that the end-to-end distance is fixed at the value $z$. Then the width averaged with respect to $z$, which we call the longitudinal boundary of the chain, is defined. A comparison is made among several dimensions determining the size of the polymer chain.
\end{abstract}

KEY WORDS Gaussian Random Coil / Diffusion Equation / Longitudinal Boundary / Maximum Extent /

In a previous $\operatorname{paper}^{1}$ (hereafter referred as I), we investigated two different widths of a Gaussian random coil chain; one was the projected length, $L$, of the whole chain along one direction taken arbitrarily in solution; the other was the diameter, $2 R$, of a circumscribing sphere of the whole chain around its center of mass. Although the diameter $2 R$ was not a maximum dimension of the chain, we were able to tell roughly the average size of the polymer chain in solution.

According to the Monte Carlo calculations by Koyama $^{2}$ and by Šolc and Stockmayer, ${ }^{3}$ a polymer chain has an asymmetric shape even if the distribution function of its end-to-end distance is given approximately by the symmetric Gaussian function. These workers calculated the average ratios of the orthogonal components of the radius of gyration tensor along the principal axes of the inertia of the unrestricted random flight chain and defined the equivalent ellipsoid of the chain. In the Koyama theory, the cubic equation for the three components of the tensor was solved by preaveraging the coefficients and from its most simplified form, an analytical solution was also obtained. By making use of this equivalent ellipsoid-polymer model and assuming the expressions for the intramolecular interactions between segments, Koyama calculated the excluded-volume effect on the radius of gyration and on the intrinsic viscosity of the chain polymer.
In the Šolc and Stockmayer theory, consideration was made of the ensemble of lattice chains where each configuration of the chain was given by a series of successive steps of random walk; the step length corresponds to the bond length of the polymer chain. These authors made a detailed investigation of the shape distribution by calculating the higher moments of the components of the tensor as well as the second moments which were in good agreement with the Koyama results. Doi and Nakajima ${ }^{4}$ also investigated this problem, using a Rouse model and obtained analytically the average values of these three principal components. They assumed that the shape of the chain is determined by the first few normal modes. The results were in good agreement with the Šolc results. ${ }^{3}$

Later, this problem was extensively investigated by Rubin and Mazur ${ }^{5,6}$ in both cases of a selfavoiding walk (a chain with excluded-volume effect) and an unrestricted walk (an unperturbed chain). The sets of spans of both the self-avoiding and the unrestricted models were measured with respect to each of the space-fixed ${ }^{5}$ and the chain-fixed ${ }^{6}$ axes. The width (or span) of a random walk in one direction $\boldsymbol{n}$ is defined as the minimum distance between parallel planes normal to $n$ which contain whole lattice points visited by the walk. The direction of the maximum span of the configuration was taken as the base of a set of orthogonal chain- 
fixed axes. ${ }^{6}$ The average axial ratios and the distribution functions of spans along the three principal directions of each of these axes were found by computer calculation, using the Monte Carlo method. Then the set of directions of the chain-fixed axes was found to be nearly the same as the set of directions of the principal components of the radius of gyration tensor by Šolc and Stockmayer. ${ }^{3}$ It was also found that the direction of the end-to-end vector is correlated to that of the maximum span of the chain configuration. Analytic solutions of the widths with respect to space-fixed axes were also obtained in the case of the unperturbed chain. ${ }^{5}$ No attempt was made to obtain analytically the average widths (or spans) with respect to the chain-fixed axes. The diameter $2 R$ obtained in I is not a suitable parameter for estimating the size of the ellipsoidal polymer chain. Furthermore, as shown in Discussion, $2 R$ is too large when it is compared with the maximum extent (or span) of the chain obtained by Rubin et al. ${ }^{6}$ The necessity of obtaining another parameter like their maximum extent and investigating the asymmetric shape of the chain with respect to chain-fixed axes led us to perform further calculations.

Hollingsworth ${ }^{7}$ obtained analytically the transverse boundary, $\rho$, of the chain which is the distance from the line connecting both ends of the chain to the segment which is most distant from the line.

In this paper, we consider the "longitudinal" boundary of the Gaussian random-coil chain which is another width measured along its end-to-end direction, $\boldsymbol{r}$, since we assume that the chain is elongated to this direction (see Figure 1). The line joining both ends is taken as a base of the set of the orthogonal axes. First, the parameter is calculated

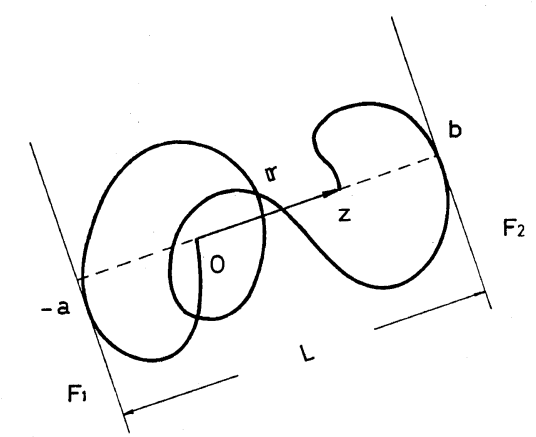

Figure 1. The width, $L$, of a polymer chain along the line joining both ends. by the diffusion-equation method developed in I when the end-to-end distance is constrained to the value $z$. This restriction is then removed, by taking an average with respect to $z$, using the radial distribution function of $z$. This new parameter averaged with respect to $z$ is defined as the longitudinal boundary. Finally, several parameters specifying the size of the polymer chain are compared.

\section{WIDTH OF A CHAIN ALONG THE LINE CONNECTING BOTH ENDS WITH CONSTANT END-TO-END DISTANCE}

Let $W(\boldsymbol{r} ; n) \mathrm{d} \boldsymbol{r}$ be the probability that the vector from one end to the other of a polymer chain of $n$ monomer units lies in the volume element $\mathrm{d} \boldsymbol{r}$ which has the position vector $r=(r, z)$. If the degree of polymerization $n$ is large, the end-to-end distance of a polymer chain obeys a Gaussian distribution function. ${ }^{8}$ Thus, the distribution function $W(\dot{r}, n)$ satisfies the following "diffusion" equation provided that $n$ is taken as a continuous variable:

$$
\frac{\partial W}{\partial n}=D \nabla^{2} W
$$

where $D=b^{2} / 6$ and $b$ is the statistical bond length. We assume that the whole chain is included in the cylinder whose axis of symmetry (i.e., $z$-axis) corresponds to the line connecting both ends. In cylindrical coordinates, eq 1 is written as

$$
\frac{\partial W}{\partial n}=D\left[\frac{\partial^{2} W}{\partial r^{2}}+\frac{1}{r} \frac{\partial W}{\partial r}+\frac{\partial^{2} W}{\partial z^{2}}\right] .
$$

Let us consider a pair of parallel planes, $F_{1}$ at $z=-a$ and $F_{2}$ at $z=b$, standing normal to the $z$ direction as shown in Figure 1. As was discussed in I, the adsorption coefficient $p$ of the chain to the planes should be $1 / 2$ since these boundary planes are virtual; nevertheless, for large $n$, the effect of adsorption of the chain by the boundary planes becomes small in evaluating the width of the chain. Therefore, we adopt for simplicity the following boundary conditions ( $p=1$, i.e., complete adsorption).

$$
W(r, z=-a ; n)=W(r, z=b ; n)=0,
$$

and 
The Widths of a Polymer Chain. II.

$$
\lim _{r \rightarrow \infty} W(r, z ; n)=0 .
$$

The initial condition is

$$
\begin{aligned}
W(r, z ; n=0) & =\delta(\boldsymbol{r}) \\
& =\frac{1}{\pi r} \delta(r) \delta(z)
\end{aligned}
$$

The solution of eq 2 under these conditions is given through Fourier series expansion of $W$ with respect to $z$ as,

$$
\begin{array}{r}
W(r, z, a, L ; n)=\frac{2}{L} \sum_{m=1}^{\infty} \sin \frac{m \pi a}{L} \sin \frac{m \pi(z+a)}{L} \\
\quad \times \exp \left[-\left(\frac{m \pi}{L}\right)^{2} D n\right] \frac{1}{4 \pi D n} \exp \left(-\frac{r^{2}}{4 D n}\right) \\
=\left(\frac{1}{4 \pi D n}\right)^{3 / 2} \sum_{l=-\infty}^{\infty}\left\{\exp \left[-\frac{(z+2 L)^{2}}{4 D n}\right]\right. \\
\left.\quad-\exp \left[-\frac{(z+2 a+2 L l)^{2}}{4 D n}\right]\right\} \exp \left(-\frac{r^{2}}{4 D n}\right),
\end{array}
$$

where $L=a+b$ is the distance between the boundary planes. In the limit of large $L$ and $a, W(r, z, a$, $L ; n)$ corresponds, of course, to the well-known Gaussian distribution function

$$
\begin{aligned}
\lim _{\substack{L \rightarrow \infty \\
a \rightarrow \infty}} W(r, z, a, L ; n) & =\left(\frac{1}{4 \pi D n}\right)^{3 / 2} \exp \left(-\frac{r^{2}+z^{2}}{4 D n}\right) \\
& \equiv W_{0}(r, z ; n)
\end{aligned}
$$

where $W_{0}(r, z ; n)$ is the solution of eq 2 subject to no boundary planes.

Let us define by $F(z, a, L ; n)=W(r, z, a, L$; $n) / W_{0}(r, z ; n)$, the fraction of chains whose width are not larger than $L$; the range of variable $a$, i.e., the distance from one end to the boundary, is $0 \leqq a \leqq L-z$ or $-z \leqq a \leqq L$, according as $z \geqq 0$ or $z<0$. The function $F(z, a, L ; n)$, when averaged with respect to $a$, is the probability that the whole chain lies completely in the region between the parallel planes. Therefore, we define the function $w(z, L ; n)$ as follows, putting $z=-z$ for $z<0$ :

$$
\begin{aligned}
w(z, L ; n)= & \frac{1}{2}\left\{\frac{1}{L-z} \int_{0}^{L-z} F(z, a, L ; n) \mathrm{d} a\right. \\
& \left.+\frac{1}{L-z} \int_{z}^{L} F(-z, a, L ; n) \mathrm{d} a\right\},
\end{aligned}
$$

$$
\begin{gathered}
=\sum_{l=-\infty}^{\infty}\left\{\exp \left[-\frac{(L l)^{2}}{D n}\right] \cosh \left(\frac{L l z}{D n}\right)\right. \\
-\frac{1}{L-z} \exp \left[\frac{z^{2}}{4 D n}\right] \int_{L l+z / 2}^{L(l+1)-z / 2} \\
\left.\quad \times \exp \left[-\frac{t^{2}}{D n}\right] \mathrm{d} t\right\} .
\end{gathered}
$$

Consequently, the probability density $f_{z}(L)$ that the width of a polymer chain along the line connecting both ends is precicely $L$ under the restriction that the end-to-end distance is fixed at the absolute value $z$ is related to $w(z, L ; n)$ as follows.

$$
\frac{1}{L-z} \int_{z}^{L}(L-l) f_{z}(l) \mathrm{d} l=w(z, L ; n) .
$$

This yields the following relation.

$$
\begin{aligned}
f_{z}(L)= & \frac{\mathrm{d}^{2}}{\mathrm{~d} L^{2}}\{(L-z) w(z, L ; n)\} \\
= & \sum_{l=-\infty}^{\infty} \frac{l}{D n}[-2 L(2 l+1)(l+1)+(L-z) \\
& \left.\times\left\{\frac{l}{D n}(2 L l+z)^{2}+2\right\}\right] \exp \left[-\frac{L l}{D n}(L l+z)\right] .
\end{aligned}
$$

Several curves of $\sqrt{n} b t f_{z}(L / z)$ are shown in Figure 2 as functions of $L / z$ for values of $t=z / \sqrt{n} b$. The first and the second moments of the width for a

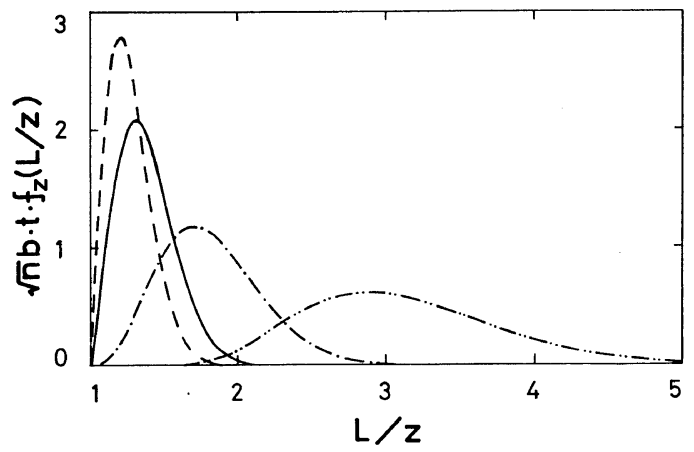

Figure 2. The probability density, $\sqrt{n} b t f_{z}(L / z)$, as a function of $L / z$ for several values of $t=\mathrm{z} / \sqrt{n} b$; a broken line for $t=1$; a solid line for $t=\sqrt{2 / 3}$, the most probable $z$, where the radial distribution function, $4 \pi z^{2} P(z)$, of $z$ takes the maximum value; a dot-dashed line for $t=1 / 2$; and dot-dot-dashed line for $t=1 / 4$. 
constrained end-to-end separation can be obtained from eq 9 as (see Appendix),

$$
\langle L\rangle_{z}=\sqrt{n} b\left[t+\sqrt{\frac{2}{3}} \exp \left(\frac{3}{2} t^{2}\right) \operatorname{Erfc}\left(\sqrt{\frac{3}{2}} t\right)\right]
$$

and

$$
\begin{array}{rl}
\left\langle L^{2}\right\rangle_{z}=n & n b^{2}\left[t^{2}+\frac{1}{2}+\frac{t}{\sqrt{6}}\right. \\
& \times \exp \left(\frac{3}{2} t^{2}\right) \operatorname{Erfc}\left(\sqrt{\frac{3}{2}} t\right) \\
+ & \frac{1}{6} \sum_{l=1}^{\infty} \frac{1}{l^{2}(l+1)^{2}} \exp \left[-6 l(l+1) t^{2}\right] \\
& -\frac{t}{\sqrt{6}} \exp \left(\frac{3}{2} t^{2}\right) \\
& \left.\times \sum_{l=1}^{\infty} \frac{2 l+1}{l^{2}(l+1)^{2}} \operatorname{Erfc}\left[\sqrt{\frac{3}{2}}(2 l+1) t\right]\right]
\end{array}
$$

where

$$
\operatorname{Erfc} x=\int_{x}^{\infty} \mathrm{e}^{-t^{2}} \mathrm{~d} t
$$

is the error function. From Figure 2 we obtain the value $L_{\mathrm{m}}$ of $L$ for the maximum of $f_{z}(L)$. In Table I, the numerical values of $\langle L\rangle_{z},\left\{\left\langle L^{2}\right\rangle_{z}\right\}^{1 / 2}$ and $L_{\mathrm{m}}$ are

\begin{tabular}{|c|c|c|c|}
\hline $\begin{array}{l}\text { End-to-end } \\
\text { distance, } \\
(\sqrt{n} b)\end{array}$ & $\begin{array}{c}\text { First } \\
\text { moment, } \\
\langle L\rangle_{z} \\
(\sqrt{n} b)\end{array}$ & $\begin{array}{l}\text { Root mean } \\
\text { square of } \\
\text { second } \\
\text { moment, } \\
\left\{\left\langle L^{2}\right\rangle_{z}\right\}^{1 / 2} \\
(\sqrt{n} b)\end{array}$ & $\begin{array}{c}\text { Most } \\
\text { probable } \\
\text { width, } \\
L_{\mathrm{m}}(\sqrt{n} b)\end{array}$ \\
\hline 2 & 2.15 & 2.16 & 2.09 \\
\hline 1 & 1.27 & 1.28 & 1.20 \\
\hline$\sqrt{2 / 3}$ & 1.13 & 1.14 & 1.06 \\
\hline $1 / 2$ & 0.91 & 0.92 & 0.85 \\
\hline $1 / 4$ & 0.78 & 0.80 & 0.72 \\
\hline $1 / 8$ & 0.74 & 0.76 & 0.68 \\
\hline
\end{tabular}
shown for each value of $z$. In the case of $z=0$, the first and the second moments are given by

$$
\langle L\rangle_{z}=\sqrt{\frac{\pi}{6}} \sqrt{n} b,
$$

Table I. The values of $\langle L\rangle_{z},\left\{\left\langle L^{2}\right\rangle_{z}\right\}^{1 / 2}$

$$
\text { and } L_{\mathrm{m}}
$$

and

$$
\begin{aligned}
\left\langle L^{2}\right\rangle_{z} & =\left\{\frac{1}{2}+\frac{1}{6} \sum_{l=1}^{\infty} \frac{1}{l^{2}(l+1)^{2}}\right\} n b^{2} \\
& =\frac{\pi^{2}}{18} n b^{2},
\end{aligned}
$$

respectively. Those values corresponds, of course, to the results for a ring polymer. ${ }^{9}$

\section{LONGITUDINAL BOUNDARY OF A POLYMER CHAIN}

The function $f_{z}(L)$, when averaged with respect to $z$, yields the probability density that the width of the chain along the line connecting both ends is precisely $L$ irrespective of the value $z$. We call this width the longitudinal boundary of the chain. We thus define the distribution function $f(L)$ as

$$
f(L)=\int_{0}^{\infty} 4 \pi z^{2} P(z) f_{z}(L) \mathrm{d} z,
$$

where $4 \pi z^{2} P(z)$ is the radial distribution function of $z$ which is the probability density that $z(=|\boldsymbol{r}|)$ lies between $z$ and $z+\mathrm{d} z$ irrespective of the direction $\boldsymbol{r}$,

$$
P(r)=W_{0}(r ; n)=\left(\frac{1}{4 \pi D n}\right)^{3 / 2} \exp \left(-\frac{r^{2}}{4 D n}\right) .
$$

Substituting eq 9 and eq 15 into eq 14, we obtain

$$
\begin{aligned}
f(L)= & \frac{2}{\sqrt{\pi D n}} \sum_{l=-\infty}^{\infty}\left[\left\{l(2 l+1) \frac{L^{2}}{D n}+4 l(4 l+1)\right\}\right. \\
& \times \exp \left[-\frac{\left(l+\frac{1}{2}\right)^{2}}{D n} L^{2}\right]-4 l(4 l+1) \\
& \times \exp \left(-\frac{l^{2}}{D n} L^{2}\right)+4 l^{2}(8 l+3) \frac{L}{D n} \int_{l L}^{(l+1 / 2) L} \\
& \left.\times \exp \left(-\frac{t^{2}}{D n}\right) \mathrm{d} t\right] .
\end{aligned}
$$

This function is shown in Figure 3. The first and the second moments of $f(L)$ are calculated in the following:

$$
\langle L\rangle=\frac{8}{3} \sqrt{\frac{2}{3 \pi}} \sqrt{n} b,
$$

and 
$\left\langle L^{2}\right\rangle=\left\{\frac{13}{8}+\frac{1}{24} \sum_{l=1}^{\infty} \frac{1}{l^{2}(l+1)^{2}(2 l+1)^{3}}\right\} n b^{2} \simeq \frac{13}{8} n b^{2}$.

The most probable value $L_{\mathrm{m}}$ of $L$, where $f(L)$ takes maximum value, is obtained from the curve as

$$
L_{\mathrm{m}}=1.130 \sqrt{n} b .
$$

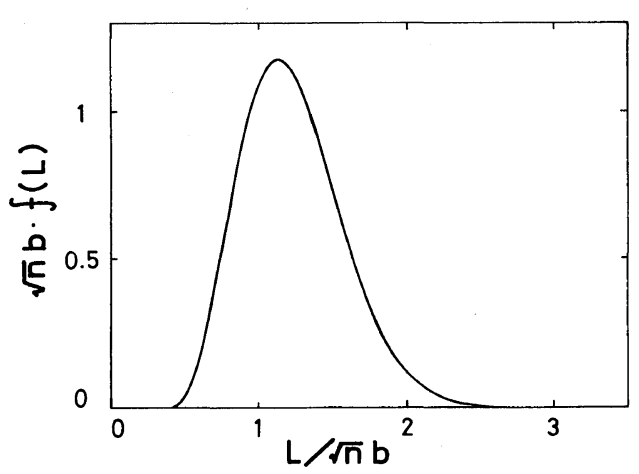

Figure 3. The probability density, $\sqrt{n} b f(L)$, as a function of $L / \sqrt{n} b$.

\section{DISCUSSION}

As mentioned in the previous section, we considered the diameter of the circumscribing sphere around the center of mass in I. We obtained the probability density $F(R)$ that the whole chain is contained in the sphere of radius $R$ around the center of mass and just makes contact with the sphere. The first and the second moments of $F(R)$ are given by

$$
\langle R\rangle=\frac{1}{6} \sqrt{\frac{\pi^{3}}{2}} \sqrt{n} b,
$$

and

$$
\left\langle R^{2}\right\rangle=\frac{7}{18} \zeta(3) n b^{2},
$$

respectively, where $\zeta(k)$ is the zeta function

$$
\zeta(k)=\sum_{n=1}^{\infty} \frac{1}{n^{k}} .
$$

Hollingsworth ${ }^{10}$ considered the radius of a circumscribing sphere around one end of the polymer chain. Later, Weidman et al. ${ }^{11}$ gave an exact expression for this radius (denoted as $R_{\mathrm{WKK}}$ ). The first and the second moments of the distribution function of $R_{\mathrm{WKK}}$ are obtained as

$$
\left\langle R_{\mathrm{WKK}}\right\rangle=\frac{1}{2} \sqrt{\frac{\pi^{3}}{6}} \sqrt{n} b,
$$

and

$$
\left\langle R_{\mathrm{WKK}}^{2}\right\rangle=\frac{7}{6} \zeta(3) n b^{2},
$$

respectively. In Table II, a comparison is made of these values and $\langle L\rangle$ and $\left\langle L^{2}\right\rangle$. The average longitudinal boundary $L$ takes an intermediate value between $R_{\mathrm{wKK}}$ and $2 R$; the parameter $L$ thus seems better for estimating the size of a polymer chain than $2 R$.

The transverse boundary $\rho$ of a random-coil chain was calculated by Hollingsworth; this is the distance of the link which is furthest from the line joining the ends of the chain. This parameter can be obtained independently of the longitudinal boundary provided that the Gaussian distribution is

Table II. Dimensions of the Random Coil Chain

\begin{tabular}{lccc}
\hline $\begin{array}{l}\text { Dimensions of the } \\
\text { random-coil chain }\end{array}$ & $\begin{array}{c}\text { Radius of the contact } \\
\text { sphere around one end, } \\
R_{\mathrm{WKK}}\end{array}$ & $\begin{array}{c}\text { Longitudinal } \\
\text { boundary, } \\
L\end{array}$ & $\begin{array}{c}\text { Diameter of the contact } \\
\text { sphere around the } \\
\text { center of mass, } 2 R\end{array}$ \\
\hline $\begin{array}{l}\text { First } \\
\text { moment } \\
(\sqrt{n} b)\end{array}$ & $\frac{1}{2} \sqrt{\frac{\pi^{3}}{6}}=1.14$ & $\frac{8}{3} \sqrt{\frac{2}{3 \pi}}=1.23$ & $\frac{1}{3} \sqrt{\frac{\pi^{3}}{2}}=1.31$ \\
\hline $\begin{array}{l}\text { Root mean square } \\
\text { of second moment } \\
(\sqrt{n} b)\end{array}$ & $\sqrt{\frac{7}{6} \zeta(3)}=1.18$ & $\sqrt{\frac{13}{8}}=1.27$ & $2 \sqrt{\frac{7}{18} \zeta(3)=1.37}$ \\
\hline
\end{tabular}


assumed for the end-to-end distance. The first and the second moments of the probability density $f(\rho)$ are given numerically by

and

$$
\langle\rho\rangle=0.62 \sqrt{n} b
$$

$$
\left\{\left\langle\rho^{2}\right\rangle\right\}^{1 / 2}=0.64 \sqrt{n} b,
$$

respectively. Then the axial ratio of the chain obtained from the values of the longitudinal boundary and the transverse boundary is

$$
2\langle\rho\rangle\langle L\rangle \simeq 1 .
$$

Let us compare the analytic results (17) and (21) with the Monte Carlo results obtained by Rubin and Mazur. ${ }^{6}$ They calculated the set of spans measured with respect to that of three principal chain-fixed axes of the chain configuration based on the direction of the maximum extent (or span, denoted as $R_{3}(N)$ ) of the configuration. The bond length of the chain corresponds to each step length. The first and the second moments of $R_{3}(N)$, i.e., $\left\langle R_{3}(N)\right\rangle / \sqrt{N}$ and $\left\{\left\langle R_{3}^{2}(N)\right\rangle / N\right\}^{1 / 2}$, varied between 1.23 and 1.28 and between 1.27 and 1.31 respectively, according to $N$, the number of steps, changing from 50 to 200. Although the longitudinal boundary is not the maximum extent, we see in Table II that a set of values of averages $\langle L\rangle$ and $\left\{\left\langle L^{2}\right\rangle\right\}^{1 / 2}$ is very close to that of $R_{3}(N)$.

The maximum transverse distance $R_{2}(N)$ measured along the direction normal to $R_{3}(N)$ was also obtained by Rubin et al. The first and the second moments of $R_{2}(N)$ were found in each case of the step length $N$, changing from 50 to 200; the value of $\left\langle R_{2}(N)\right\rangle / \sqrt{N}$ varied between 0.73 and 0.78 ; and that of $\left\{\left\langle R_{2}^{2}(N)\right\rangle / N\right\}^{1 / 2}$ varied between 0.75 and 0.80 . No value for these averages correspond to the analytical results (21). Furthermore, the ratio of $\left\langle R_{3}(N)\right\rangle$ and $\left\langle R_{2}(N)\right\rangle$ is 1.64 approximately independent of $N$. More works are needed to obtain analytically a reasonable transverse width of the polymer chain.

\section{APPENDIX}

The first moment of $f_{z}(L)$ is defined as

$$
\langle L\rangle_{z}=\int_{z}^{\infty} L f_{z}(L) \mathrm{d} L .
$$

Substituting eq 9 into eq A1, we obtain

$$
\begin{aligned}
\langle L\rangle_{z} & =\int_{z}^{\infty} L \frac{\mathrm{d}^{2}}{\mathrm{~d} L^{2}}\{(L-z) w(z, L ; n)\} \mathrm{d} L \\
& =\left[L\{(L-z) w(z, L ; n)\}^{\prime}-(L-z) w(z, L ; n)\right]_{L=z}^{L=\infty} \\
& =z+2 \exp \left(\frac{z^{2}}{4 D n}\right) \int_{z / 2}^{\infty} \exp \left(-\frac{t^{2}}{D n}\right) \mathrm{d} t . \quad \text { (A2) }
\end{aligned}
$$

Putting $t=z / \sqrt{n} b$ and $D=b^{2} / 6$, we have

$$
\langle L\rangle_{z}=\sqrt{n} b\left[t+\sqrt{\frac{2}{3}} \exp \left(\frac{3}{2} t^{2}\right) \operatorname{Erfc}\left(\sqrt{\frac{3}{2}} t\right)\right]
$$

where Erfc is the error function

$$
\operatorname{Erfc} x=\int_{x}^{\infty} \mathrm{e}^{-t^{2}} \mathrm{~d} t .
$$

The second moment is also defined as

$$
\begin{aligned}
\left\langle L^{2}\right\rangle_{z}= & \int_{z}^{\infty} L^{2} f_{z}(L) \mathrm{d} L, \\
= & {\left[L^{2} \frac{\mathrm{d}}{\mathrm{d} L}\{(L-z) w(z, L ; n)\}\right.} \\
& -2 L(L-z) w(z, L ; n)]_{L=z}^{L=\infty} \\
& +2 \int_{z}^{\infty}(L-z) w(z, L ; n) \mathrm{d} L .
\end{aligned}
$$

The first term of eq A4 is rewritten as

$$
\begin{gathered}
{\left[L^{2} \frac{\mathrm{d}}{\mathrm{d} L}\{(L-z) w(z, L ; n)\}-2 L(L-z) w(z, L ; n)\right]_{L=z}^{L=\infty}} \\
=\lim _{L \rightarrow \infty}\left[-L^{2}+2 L z+4 L \exp \left(\frac{z^{2}}{4 D n}\right)\right. \\
\left.\quad \times \int_{z / 2}^{\infty} \exp \left(-\frac{t^{2}}{D n}\right) \mathrm{d} t\right] .
\end{gathered}
$$

The second term of eq A4 is given by

$$
\begin{aligned}
& 2 \int_{z}^{\infty}(L-z) w(z, L ; n) \mathrm{d} L \\
& =\lim _{L \rightarrow \infty}\left[L^{2}-2 L z-4 L \exp \left(\frac{z^{2}}{4 D n}\right)\right. \\
& \left.\quad \times \int_{z / 2}^{\infty} \exp \left(-\frac{t^{2}}{D n}\right) \mathrm{d} t\right]+z^{2}+3 D n
\end{aligned}
$$

Polymer J., Vol. 13, No. 2, 1981 


$$
\begin{aligned}
& +z \exp \left(\frac{z^{2}}{4 D n}\right) \int_{z / 2}^{\infty} \exp \left(-\frac{t^{2}}{D n}\right) \mathrm{d} t \\
& +D n \sum_{l=1}^{\infty} \frac{1}{l^{2}(l+1)^{2}} \exp \left[-\frac{l(l+1)}{D n} z^{2}\right] \\
& -z \exp \left(\frac{z^{2}}{4 D n}\right) \sum_{l=1}^{\infty} \frac{2 l+1}{l^{2}(l+1)^{2}} \\
& \quad \times \int_{(l+1 / 2) z}^{\infty} \exp \left(-\frac{t^{2}}{D n}\right) \mathrm{d} t .
\end{aligned}
$$

The first term of eq A6 diverges as $L \rightarrow \infty$, however, it cancels with eq $\mathrm{A} 5$, and we obtain

$$
\begin{aligned}
\left\langle L^{2}\right\rangle_{z}=z^{2} & +3 D n+z \exp \left(\frac{z^{2}}{4 D n}\right) \int_{z / 2}^{\infty} \exp \left(-\frac{t^{2}}{D n}\right) \mathrm{d} t \\
& +D n \sum_{l=1}^{\infty} \frac{1}{l^{2}(l+1)^{2}} \exp \left[-\frac{l(l+1)}{D n} z^{2}\right] \\
& -z \exp \left(\frac{z^{2}}{4 D n}\right) \sum_{l=1}^{\infty} \frac{2 l+1}{l^{2}(l+1)^{2}} \\
& \times \int_{(l+1 / 2) z}^{\infty} \exp \left(-\frac{t^{2}}{D n}\right) \mathrm{d} t
\end{aligned}
$$

Finally, we have

$$
\begin{aligned}
\left\langle L^{2}\right\rangle_{z}=n b^{2}\left[t^{2}\right. & +\frac{1}{2}+\frac{t}{\sqrt{6}} \\
& \times \exp \left(\frac{3}{2} t^{2}\right) \operatorname{Erfc}\left(\sqrt{\frac{3}{2}} t\right) \\
+ & \frac{1}{6} \sum_{l=1}^{\infty} \frac{1}{l^{2}(l+1)^{2}} \exp \left[-6 l(l+1) t^{2}\right] \\
& -\frac{t}{\sqrt{6}} \exp \left(\frac{3}{2} t^{2}\right) \sum_{l=1}^{\infty} \frac{2 l+1}{l^{2}(l+1)^{2}} \\
& \left.\times \operatorname{Erfc}\left[\sqrt{\frac{3}{2}}(2 l+1) t\right]\right] .
\end{aligned}
$$

\section{REFERENCES}

1. K. Moro and N. Saito, Polym. J., 8, 377 (1976).

2. R. Koyama, J. Phys. Soc. Jpn., 22, 973 (1967); ibid., 24, 580 (1968).

3. K. Šolc and W. H. Stockmayer, J. Chem. Phys., 54, 2756 (1971); K. Šolc, ibid., 55, 335 (1971); K. Šolc, W. H. Stockmayer, and W. Gobush, Macromolecules, 8 , 690 (1975).

4. M. Doi and H. Nakajima, Chem. Phys., 6, 124 (1974).

5. R. J. Rubin and J. Mazur, J. Chem. Phys., 63, 5362 (1975).

6. R. J. Rubin and J. Mazur, Macromolecules, 10, 139 (1977).

7. C. A. Hollingsworth, J. Chem. Phys., 17, 97 (1949).

8. H. Yamakawa, "Modern Theory of Polymer Solutions," Harper \& Row, New York, N.Y., 1971.

9. R. J. Rubin, J. Mazur, and G. H. Weiss, Pure Appl. Chem., 46, 143 (1976).

10. C. A. Hollingsworth, J. Chem. Phys., 16, 544 (1948).

11. J. J. Weidman, H. Kuhn and W. Kuhn, J. Chim. Phys., 50, 226 (1953). 